\title{
Aspergillus Calidoustus Endocarditis in a Child after Cardiac Surgery: Case Report
}

\author{
Laura A Broughton ${ }^{1 *}$, Robert A Broughton ${ }^{2}$ and Abeer H Hamdy ${ }^{3}$ \\ ${ }^{1}$ Heinrich A. Werner Division of Pediatric Critical Care, University of Kentucky, USA \\ ${ }^{2}$ Division of Pediatric Infectious Diseases, University of Kentucky, USA \\ ${ }^{3}$ Division of Pediatric Cardiology, University of Kentucky, USA
}

*Corresponding author: Laura A Broughton, First Author, 800 Rose Street, MN 460, Lexington, KY 40536, USA.

Received Date: February 17, 2021

Published Date: March 16, 2021

\begin{abstract}
We report a case of a 14-year-old male with Aspergillus calidoustus endocarditis occurring in an expanded polytetrafluoroethylene conduit for Tetralogy of Fallot. Aspergillus calidoustus is a concerning newly described species that is often resistant to voriconazole. Our patient was optimally treated by using antifungal therapy active against the fungus and surgical resection of the infected tissue.

Keywords: Aspergillus calidoustus; Fungal endocarditis; Voriconazole; Amphotericin B

Abbreviations: RVOT: Right ventricular outflow tract; ePTFE: expanded polytetrafluoroethylene; NT-proBNP: N-terminal pro b-type natriuretic peptide; RV-PA: Right ventricular to pulmonary artery; CTA: Computed tomography angiogram; MIC: Minimum inhibitory concentration; GMS: Gomori methenamine silver
\end{abstract}

\section{Introduction}

Fungal endocarditis is relatively rare with an overall poor prognosis [1]. Aspergillus endocarditis is a severe form of fungal endocarditis, accounting for $20-30 \%$ of all fungal endocarditis cases [2]. Aspergillus calidoustus is a newly described species identified as being distinct from Aspergillus ustus in 2008 [3]. It is an emerging pathogen that is concerning as it is more resistant to antifungals, especially azoles, than other species of Aspergillus [3]. We report a rare case of endocarditis due to Aspergillus calidoustus in an immunocompetent 14-year-old male after cardiac surgery. After surgical resection of infected tissue and 17 months of antifungal therapy, his clinical outcome was favorable.

\section{Case Presentation}

A 14-year-old male with a history of Tetralogy of Fallot, corrected with a transannular patch as a neonate, underwent a right

ventricular outflow tract (RVOT) reconstruction using an expanded polytetrafluoroethylene (ePTFE) conduit. His intraoperative and postoperative courses were uncomplicated.

He was readmitted three months later with a one-month history of fever, cough, 21-pound weight loss, and dyspnea with exertion. On physical exam, he was pale appearing but in no acute distress. Vital signs on admission included heart rate: 130 beats/ min, respiratory rate: 29 breaths/min, blood pressure: 118/77 $\mathrm{mmHg}$, and temperature: $38.1^{\circ} \mathrm{C}$. Initial laboratory values revealed a slight leukocytosis (13.72 k/uL), elevated C-reactive protein (16.4 $\mathrm{mg} / \mathrm{dL}$ ), and elevated $\mathrm{N}$-terminal pro b-type natriuretic peptide (NT- proBNP) (3456pg/mL). A transthoracic echocardiogram revealed an echogenic area at and just above the pulmonary valve of the right ventricular to pulmonary artery (RV-PA) conduit, trace pulmonary insufficiency, moderate pulmonary stenosis, moderate 
RV enlargement with moderate hypertrophy and moderately depressed RV function. Three large volume blood cultures were obtained, and vancomycin and ceftriaxone were initiated for presumed endocarditis. At 48 hours' incubation, blood cultures remained negative and thus treatment for suspected culture negative endocarditis was transitioned to vancomycin, cefepime, rifampin, and gentamicin. Fungal blood cultures were also sent at this time, which were ultimately negative. However, he had persistently elevated inflammatory markers and his clinical status worsened with increasing tachycardia, diaphoresis, and dyspnea on exertion. A repeat transthoracic echocardiogram on day 7 of admission demonstrated a worsening gradient across the RV-PA conduit. A chest coronary computed tomography angiogram (CTA) was performed which demonstrated a significant thrombus versus vegetation in the RV-PA conduit with $60-70 \%$ stenosis in addition to bilateral pulmonary emboli. On day 8 of hospitalization, the patient was taken to the cardiac catheterization lab for Angiojet catheter direct lysis of the thrombus/vegetation. Although unsuccessful, a tissue sample was obtained and sent for bacterial and fungal cultures. A KOH preparation of the tissue revealed septate hyphae. The same day, (1,3)-beta-D-glucan was resulted as $>500 \mathrm{pg} / \mathrm{mL}$ (negative $<80 \mathrm{mg} / \mathrm{mL}$ ) and aspergillus galactomannan index as 3.498 (negative $<0.5$ ). At this time, antifungal therapy was initiated with intravenous voriconazole $(6 \mathrm{mg} / \mathrm{kg}$ q $12 \mathrm{hx} 2$ loading doses, followed by $4 \mathrm{mg} / \mathrm{kg} \mathrm{q} 12 \mathrm{~h}$ ) and micafungin (150 mg daily). On day 10 , the patient was taken to the operating room for replacement of the RV-PA conduit (Figure 1) with a $25 \mathrm{~mm}$ pulmonary homograft. Aspergillus ustus was originally identified from the vegetation at our institution. However, his organism was sent to a specialty fungal laboratory for confirmation of speciation and susceptibility testing, which later identified the species as Aspergillus calidoustus that was resistant to voriconazole with a minimum inhibitory concentration (MIC) of $4 \mathrm{mg} / \mathrm{mL}$, but susceptible to amphotericin $\mathrm{B}$, micafungin, and terbinafine. Antifungal therapy was changed to monotherapy with liposomal amphotericin B ( $3 \mathrm{mg} / \mathrm{kg}$ daily) and he was discharged on day 28 of hospitalization. Three weeks later micafungin was restarted due to a rise then slow decline in his galactomannan antigen index and the presence of new pulmonary nodules when compared to his original chest CT. He completed a 12-week course of liposomal amphotericin B and a 9-week course of micafungin prior to transitioning to oral terbinafine $(250 \mathrm{mg}$ daily) which was continued for 14 months. He was clinically doing well with normalized inflammatory markers and resolution of his elevated serum galactomannan index when it was last measured 1 month following the discontinuation of antifungal therapy.

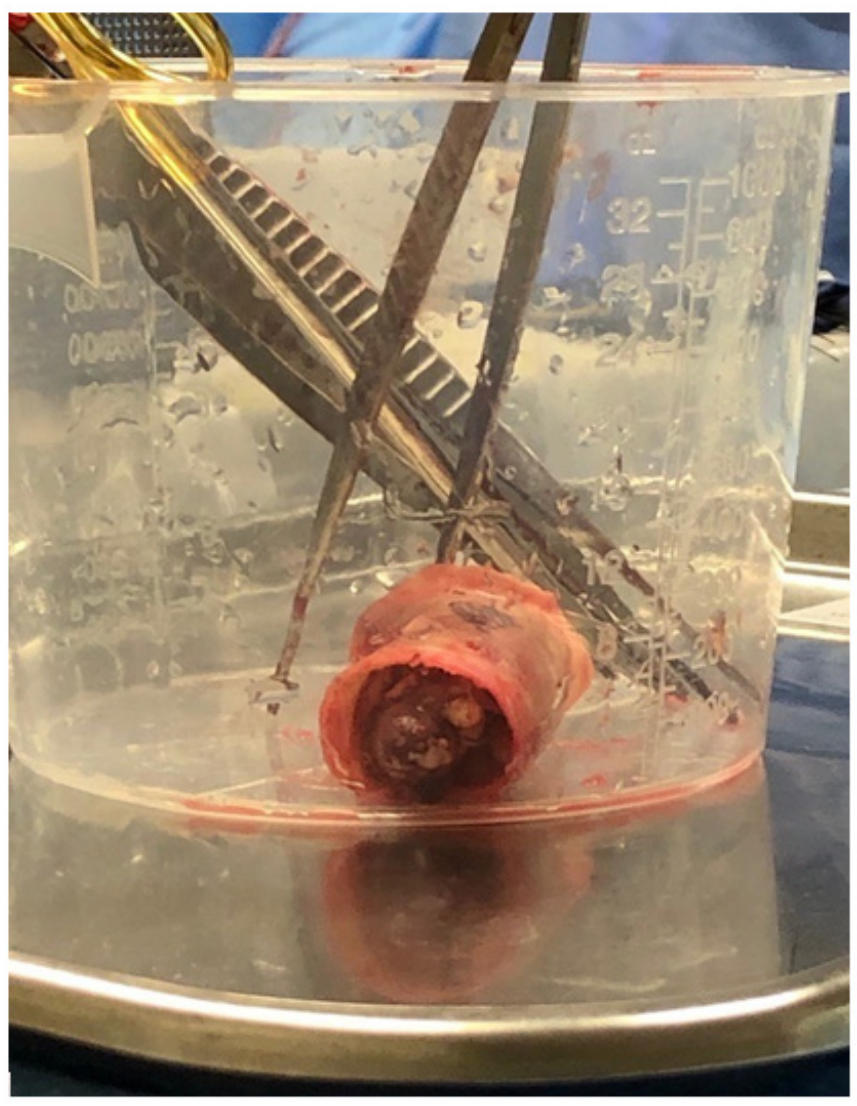

Figure 1: Fungal vegetation with thrombus inside RV-RA conduit. 


\section{Discussion}

Fungal endocarditis caused by Aspergillus is a rare disease with a high mortality rate and is associated with multiple morbidities4. In children, congenital heart disease is the most common risk factor for its development, but other risk factors include immunosuppression, intravenous drug abuse, prolonged antibiotic therapy, and parenteral nutrition [1,2]. Aspergillus endocarditis is associated with large vegetations, most commonly associated with the mitral or aortic valve and less frequently with the tricuspid and pulmonic valve [2]. An RV-PA conduit using ePTFE material places a patient at increased risk for infection, however, these conduits have been known to be an appealing alternative to homograft conduits due to their lack of potential sensitization [5].

One study indicated that only $32 \%$ of patients survive the acute episode of aspergillus endocarditis and the diagnosis was made post-mortem in $21 \%$ of the cases 2 . This is thought to be related in part to difficulty of establishing the diagnosis. Even though our patient had fever, fever may be absent in aspergillus endocarditis, having been reported to be present in only $67.7 \%$ of cases4. Fungal blood cultures are often negative, as they were in our patient. 1 The sensitivity of detecting Candida species in blood cultures is approximately 50-70\%, whereas for Aspergillus species it is only $4 \%$ [1]. The measurement of $(1,3)$-beta-D-glucan may be a useful adjunctive test to establish the diagnosis of invasive fungal disease. (1,3)-beta-D-glucan is a polysaccharide present in the cell wall of most, but not all, fungi. It has a sensitivity and specificity in patients with invasive aspergillosis of $81 \%$ and $82 \%$, respectively [6]. Aspergillus galactomannan antigen is present in the cell wall of Aspergillus and is detected when it is released during proliferation. It has a sensitivity and specificity in patients with invasive aspergillosis of $49 \%$ and $97 \%$, respectively [6]. Thus, the galactomannan antigen is more specific, but the $(1,3)$-beta-Dglucan is more sensitive [6]. Ultimately, confirmatory diagnosis usually requires obtaining tissue for histology and fungal culture to allow identification of the infecting species and susceptibility testing. Histology usually demonstrates septate hyphae on Gomori methenamine silver (GMS) staining or $\mathrm{KOH}$ preparation [2]. Although Aspergillus ustus was initially identified from the tissue culture of our patient, the isolate was sent to a special laboratory for confirmation of species identification and susceptibility testing due to its clinical significance and the organism was identified as Aspergillus calidoustus. To our knowledge, this is the first report of fungal endocarditis caused by Aspergillus calidoustus. This organism is an emerging pathogen that was described as being distinct species in 2008, as it is able to grow at or above 37 degrees Celsius, unlike Aspergillus ustus and Aspergillus granulosus [3].

Optimal treatment of aspergillus endocarditis centers on antifungal therapy active against the fungus and surgical resection of infected tissue. Surgical resection of the infected valve is mandatory, as one study demonstrated only $4 \%$ of cases were treated successfully with antifungal therapy alone [2]. Our patient was taken to the operating room for removal of the infected conduit within 36 hours of knowing that he was likely infected with an Aspergillus species. In addition to surgical resection, antifungal therapy should be initiated with intravenous voriconazole or a lipid formulation of amphotericin B [7]. We elected to initially use voriconazole in our patient as it is active against most species responsible for invasive aspergillosis, its use has been associated with improved outcomes in patients with invasive aspergillosis in comparison with amphotericin B, and it is associated with less nephrotoxicity, less electrolyte disturbances, and no infusionrelated side effects in comparison to amphotericin B [2]. Although not routinely recommended, we also added micafungin due to it being a fungicidal agent and for its potential additive or synergistic activity with voriconazole against Aspergillus species. However, voriconazole often has reduced activity against Aspergillus calidoustus. The isolate from our patient demonstrated a minimum inhibitory concentration (MIC) of voriconazole against the isolate of $4 \mathrm{mg} / \mathrm{mL}$. There are no national clinical laboratory standards for interpretation of these MICs in the United States, but the European literature suggests a voriconazole MIC $>2 \mathrm{mg} / \mathrm{mL}$ to be considered to be resistant [8]. The isolate from our patient was susceptible to amphotericin B, micafungin, and terbinafine. He was transitioned to monotherapy with liposomal amphotericin B when the antifungal susceptibilities were known. His aspergillus galactomannan index had become negative within two weeks following initiation of antifungal therapy and surgical resection of his infected conduit, likely reflecting the large reduction of his fungal burden with the surgical resection; however, it became elevated again and then fell slower than we anticipated. At that time, a repeat chest CT scan was done which revealed no vegetation/ thrombus in his homograft but there were new nodules present bilaterally in his lungs.

Micafungin was added to the liposomal amphotericin B for its fungicidal activity and its potential additive or synergistic activity with amphotericin B. He completed a 12-week course of liposomal amphotericin B and a 9-week course of micafungin prior to transitioning to oral terbinafine, the only oral antifungal to which the organism demonstrated susceptibility. Four months after transitioning to oral terbinafine, repeat chest CT revealed continued resolution of the radiographic abnormalities that were noted earlier, and his echocardiogram revealed no residual vegetation or thrombus. When he was last seen by the infectious diseases service he was doing very well clinically. His oral terbinafine was discontinued 2 weeks later after 14 months of terbinafine therapy and a repeat aspergillus galactomannan index obtained 1 month following the discontinuation of all antifungal therapy was negative.

Why this patient developed endocarditis with this unusual pathogen is unknown, although the existence of prosthetic conduit 
material placed him at risk. There had been no other cases of postoperative Aspergillus endocarditis noted in patients undergoing cardiac surgery at this institution before or after his conduit insertion. He underwent an evaluation of his immunocompetence which was normal. He reportedly lived in a house with mold growing on the walls in his bathroom and we speculated that perhaps it was acquired from this source, but this is not known with certainty as no cultures were obtained from the house.

Fungal endocarditis due to Aspergillus continues to be a diagnostic challenge. Adjunctive tests can be used to help identify fungal endocarditis with negative fungal blood cultures but have limitations of sensitivity and specificity. Establishment of the specific microbiologic etiology and antifungal susceptibility testing is essential and usually requires obtaining infected tissue for histologic examination, fungal culture, and susceptibility testing. Most invasive aspergillus infections are caused by species that are sensitive to voriconazole. However, voriconazole often has reduced activity against Aspergillus calidoustus, as was seen in our patient. Optimal treatment of aspergillus endocarditis centers on antifungal therapy active against the fungus and surgical resection of infected tissue.

\section{Acknowledgement}

None.

\section{Conflict of Interest}

No conflict of interest.

\section{References}

1. Pasha AK, Lee JZ, Low SW, Desai, H, Lee KS, et al. (2016) Fungal endocarditis: Update on diagnosis and management. Am J Med 129(10): 10371043.

2. Kalokhe AS, Rouphael N, El Chami MF, Workowski KA, Ganesh G, et al. (2010) Aspergillus endocarditis: a review of the literature. Int J Infect Dis 14(12): e1040-e1047.

3. Varga J, Houbraken J, Van Der Lee HAL, Verweij PE, Samson RA (2008) Aspergillus calidoustus sp. nov., causative agent of human infections previously assigned to aspergillus ustus. Eukaryot Cell 7(4): 630-638.

4. Meshaal MS, Labib D, Said J, Hosny M, Hassan M, et al. (2018) Aspergillus endocarditis: Diagnostic criteria and predictors of outcomes, a retrospective cohort study. PLoS One 13(8): e0201459.

5. Mercer CW, West SC, Sharma MS, Yoshida M, Morell VO (2018) Polytetrafluoroethylene conduits versus homografts for right ventricular outflow tract reconstruction in infants and young children: An institutional experience. J Thorac Cardiovasc Surg 155(5): 2082-2091.

6. Sulahian A, Porcher R, Bergeron A, Touratier S, Raffoux E, et al. (2014) Use and limits of (1-3)- $\beta$-D-Glucan assay (Fungitell), compared to galactomannan determination (Platelia Aspergillus), for diagnosis of invasive aspergillosis. J Clin Microbiol 52(7): 2328-2333.

7. Patterson TF, Thompson GR, Denning DW, Fishman JA, Hadley S, et al. (2016) Practice guidelines for the diagnosis and management of Aspergillosis: 2016 update by the Infectious Diseases Society of America. Clin Infect Dis 63(4): e1-e60

8. Ullmann AJ, Aguado JM, Arikan-Akdagli A, Denning DW, Groll AH, et al. (2018) Diagnosis and management of aspergillus diseases: executive summary of the 2017 ESCMID-ECMM-ERS guideline. Clin Microbiol Infect 24: e1-e38. 